\title{
Evaluation of General and Specific Combining Ability for Doubled Haploid Lines in Sweet Pepper (Capsicum Annuum L.)
}

Azam Zare Bayati

Azad University of Karaj

Mehran E. Shariatpanahi ( $\sim$ m_shariatpanahi2002@yahoo.com )

Agricultural Biotechnology Research Institute of Iran(ABRII) https://orcid.org/0000-0001-9634-6716

Khodadad Mostafavi

Azad University of karaj

Seied Mehdi Miri

Azad University of karaj

\section{Research Article}

Keywords: Sweet pepper, Doubled haploid, Diallel, Gene effect.

Posted Date: February 8th, 2022

DOI: https://doi.org/10.21203/rs.3.rs-1249472/v1

License: () (1) This work is licensed under a Creative Commons Attribution 4.0 International License. Read Full License 


\section{Abstract}

Hybrid cultivars are commercially and economically important; hence, too much effort needed to improve the quality and quantity of hybrids. In this research project, general (GCA) and specific (SCA) combining ability of 32 doubled haploid (DH) lines of sweet pepper produced via microspore embryogenesis were evaluated. The DH lines were crossed with the top cross parent as the tester to evaluate general combining ability (GCA). Traits including morphological and yield-related characteristics to assess the general combining ability of lines were recorded and analyzed. The half-diallel mating crosses were performed using GCA based on the selected seven DH lines for SCA evaluation. Mean squares of GCA and SCA values were highly significant for most of the characters. DH55 line indicated the highest positive GCA for average yield per plant, moreover, the highest positive SCA was obtained for $55 \times 64$ hybrid. The ratio of $\frac{M S \text { ( } G C A \text { ) }}{M S \text { ( } S C A \text { ) }}$ showed the non-additive gene actions in controlling all traits except fruit width and length and seed fresh weight. According to the results of specific combining ability of DH lines, four F1 hybrids were selected for new cultivar registration. The results obtained in the present study are applicable to release new F1-hybrids, which can be more reliable than those hybrids introduced by conventional selfing programs in sweet pepper due to $100 \%$ genetic purity of $\mathrm{DH}$ lines.

\section{Introduction}

Sweet pepper (capsicum annuum L.) is an important remunerative vegetable with a high nutritional value throughout the world belongs to the family Solanaceae used fresh or dried. This plant is diploid and has $2 n=2 x=24$ chromosomes (Jindal et al., 2019). Pepper has an international market that is consecutively increasing and world production has expanded greatly over the last decades.

One of the goals of sustainable agriculture is to enhance new strong cultivars. Hybridization program is an essential method to evolve the plants as an origin of some new genetic combinations or as a tool of speciation (Rêgo and Rêgo, 2016). To achieve this goal, one of the most important parameters is the selection of desired parents in every successful breeding program for creation of new hybrids with high yields. Diallel crossing, involves crossing parents in all possible combinations to generate hybrids (Griffing, 1956) Through which information can be obtained to heritability, types of gene actions involved in the expression of traits and predicting the performance of progeny in recent segregating generations (Syukur et al., 2010). Recognition of superior crosses requires the survey of parental general and specific combining ability to reveal the nature of gene action in the expression of traits. Accordingly, the vision of combining ability of parents and crosses and the gene effects of different traits is requisite to improve the sweet pepper.

Access to completely pure lines is a prerequisite for $F_{1}$ hybrid breeding program. On the one hand, the production of inbred lines through conventional breeding programs requires several generations of selfing. On the other hand, the procedure of inbred development is complicated because of existence of high inbreeding depression. Production of doubled haploids (DHs) is the fastest way to create quite homozygous lines in a short time. Thus using DH technology and the study of the inbred lines obtained from this method can lead to introduce strong hybrid cultivars.

According to literature and our knowledge, exploitation of haploidy system in diallel analysis of sweet pepper has not been studied (Kaushik and Dhaliwal, 2018; Kaushik, 2019a; Kaushik, 2019b).

Hereupon, this study was conducted to investigate general and specific combining abilities of the DH lines of sweet pepper, derived from microspore culture system, to achieve the $\mathrm{DH}$ parental lines for being used in $\mathrm{F}_{1}$ hybrid breeding program followed by introducing new hybrid cultivars and improving the quality of Capsicum annuum.

\section{Materials And Methods}

\section{Plant materials and production of doubled haploid (DH) lines}

All experiments were performed between 2018 and 2021. Commercial hybrid seeds including Magno (Enza Zaden), Mars, Wizard (Seminis) and Inspiration (Rijk zwaan) were planted in the greenhouse of Agricultural Biotechnology Research Institute of Iran (ABRII) as anther donor plants in cultivation trays. After 20 days, donor plantlets were transferred in main pots in a mixture of soil, peat and perlite in the ratio of $2: 1: 1(\mathrm{v} / \mathrm{v} / \mathrm{v})$ with the average temperature of $23^{\circ} \mathrm{C}$ under natural and LED light conditions (16 light/8 dark photoperiods).

Flower buds were excised early morning with a 3.5-4.0 mm length (Rodeva et al., 2007) or when the corolla was in the same size or slightly longer than calyx (Supena and Custers, 2011; Çömlekçioğlu and Ellialtıoğlu, 2018), which were at uninuclear and early binuclear developmental stage of microspores.

The flower buds at first kept in $2 \%$ sodium hypochlorite and one drop of Tween-20 solution for 10 minutes. Then the buds rinsed $3-4$ times with sterile distilled water. Based on our previously reported protocol (Heidari-Zefreh et al., 2018), the isolation of microspores and embryogenesis induction were carried out. Chromosome doubling and creation of doubled haploid lines were performed by application of colchicine in the regenerated haploid seedlings (Gil-Humanes and Barro, 2009; Esin, et al., 2016). The ploidy level of microspores-derived lines was detected using flow cytometry analysis.

\section{Top-cross test}

Identification of favorable doubled haploid (DH) lines conducted using the top-cross analysis. Thirty-two doubled haploid lines were crossed in a glasshouse with the California wonder cultivar to evaluate general combining ability (GCA). California wonder is one of the earliest open pollinated bell pepper cultivars that is the most important for improvement of pepper because of large size, favorable form, uniformity, thickness and firm flesh (Boswell, 1937). DH lines were used as maternal parents and California wonder applied as a paternal tester parent. The harvested F1 offspring were planted in a greenhouse. Only 27 doubled haploid lines led to the successful production of top-cross progeny from the crossing of the lines with the tester. The evaluation of general combining 
ability (GCA) of DH lines was conducted for morphological and yield traits, such as days to germination, flowering and fruit set, fruit fresh and dried weight, length, width and thickness of fruit diameter, number of seeds, average yield per plant, number of fruits per plant and plant height. The seven DH lines with better GCA for previously mentioned traits were selected to determine their specific combining ability through diallel cross analysis and used as superior parental lines in future F1 hybrid breeding programs.

\section{Diallel crosses method}

The seeds of top-cross picked from superior $\mathrm{DH}$ lines were collected and grown in the greenhouse. All the possible crosses were done among seven superior parental DH lines under protected greenhouse conditions in a half-diallel mating design to create twenty-one F1 hybrids. According to Möhring et al., (2011), when the parents are completely inbred (DH), reciprocal crosses and selfed parents could be omitted from the analyses, so the diallel cross "Griffing's model II method 4" was chosen (Griffing, 1956). On the other hand, the half-diallel design is more controllable, in comparing to full diallel mating scheme (Griffing, 1956). Twenty-one diallel F1 hybrids were planted in a completely randomized design (CRD) with three replications under aforementioned greenhouse conditions. Plant cultivation was done as mentioned above and also morphological, and yield characteristics on 10 randomly chosen fruits in every entry over the replications including days to germination, flowering and fruit set, fruit fresh and dried weight, length, width and thickness of fruit diameter, number of seeds, average yield per plant, number of fruits per plant, main stem length, main stem diameter and plant height were measured.

\section{Data analysis}

The analysis of variance (ANOVA) and means comparison (Duncan's multiple range means) test were performed at $5 \%$ of probability level, using SAS ${ }^{\circ}$ software (SAS Institute Inc., Cary, NC). The estimation of effects of general and specific combining ability (GCA and SCA) was calculated by SAS ${ }^{8}$ software to Griffing's method 4. The used model in the SAS software was:

$$
x_{i j k}=\mu+g_{i}+g_{j}+s_{i j}+b_{k}+e_{i j k}
$$

\section{$(i=j=1 \ldots p ; k=1 \ldots b)$}

$\mu=$ the population mean.

$g_{i}=$ the general combining ability effect of the $i$ th parent.

$g_{j}=$ the general combining ability effect of the $j$ th parent.

$S_{i j}=$ the specific combining ability effect of the cross between $i$ th and $j$ th parents.

$e_{i j k}=$ the environmental effect associated with $i j k$ th observation.

By dividing the mean squares of general on the specific combining ability and testing it according to $\mathrm{F}$ table determines the type of effects of genes as follow:

$$
\text { Effectofgene }=\frac{\mathrm{MS}(\mathrm{GCA})}{\mathrm{MS}(\mathrm{SCA})}
$$

MS (GCA): The mean square of general combining ability.

MS (SCA): The mean square of specific combining ability.

Additive and dominance variances were calculated using the following formula:

$$
\sigma^{2} G C A=\frac{1+F}{4}\left(\sigma^{2} A\right)
$$

$\sigma^{2} A$ : Additive variance

$$
\sigma^{2} G C A=\frac{1+F}{2}\left(\sigma^{2} D\right)
$$

$\sigma^{2} D$ : Dominance variance

The parental lines were fully inbred, hence in assessment of additive and dominance effect's variances, the inbreeding coefficient was considered one ( $F=1)$ (Dezfouli et al., 2019).

Private heritability was estimated using the following formula:

$$
h_{n s}^{2}=\frac{\sigma^{2} A}{\sigma^{2} P}
$$

$h_{n s}^{2}:$ Narrow-sense heritability.

$\sigma^{2} P$ : Phenotypic variance. 


\section{Results}

\section{ANOVA and mean comparison analysis}

Results of ANOVA for the studied traits were shown in Table 1. The genotype effect in the most of F1 hybrids based on the estimated traits were significant at $1 \%$ or $5 \%$ probability level (Table 1) that indicated the high diversity of the studied hybrids. Based on this study, days to germination, flowering and fruit set, length, width and diameter of fruit flesh, fruit volume, fresh and dry weight of fruit, number of lobes per fruit, length of main stem, average fruit yield per plant were significant at $1 \%$ probability level but the number of fruits per plant was significant at $5 \%$ probability level. Also, number of days to harvesting, seed number, fresh and dry weight of seeds, main stem diameter and plant height were not significant (Table 1). Means comparison analysis using Duncan's multiple range test showed that the hybrid of $\mathrm{DH} 217 \times \mathrm{DH} 218$ for Average yield/plant, fresh seed weight and dry seed weight had the highest mean (Table 2), whereas the largest number of seeds belonged to the hybrid $\mathrm{DH} 64 \times \mathrm{DH} 217$. The highest means of fresh and dry fruit weight and fruit volume were related to the cross of $\mathrm{DH} 55 \times \mathrm{DH} 53$, while the lowest amount of these traits belonged to the DH57×DH53 hybrid. The hybrid of DH55×DH64 for the lobes number per fruit and number of fruits per plant had a highest value, however there was no significant difference between this hybrid and $\mathrm{DH} 217 \times \mathrm{DH} 218, \mathrm{DH} 53 \times \mathrm{DH} 217$, DH55×DH217, DH55×DH57, DH90×DH218, DH55×DH53 and DH53×DH90 for number of fruit per plant (Table 2). The lowest number of days to germination belonged to the hybrid resulting from the crosses of DH55×DH64, DH55×DH57 and DH217×DH90 (Table 2). Although the lowest mean values of number of days to flowering were observed in a DH217×DH90 and DH53×DH218 hybrids, the hybrids DH55×DH53 and DH64×DH218 showed the least number of days to fruit set (Table 2), which indicated the early maturity of these hybrids. The highest fruit length was related to the hybrid resulting from the cross of DH64×DH57, while the maximum fruit width belonged to the hybrid DH217×DH57. In addition, the results of DH90×DH218 hybrid with an average of 6.67 mm had the highest flesh diameter (pericarp) of fruit (Table 2). As shown in Table 2, no hybrids had significant difference in terms of days to fruit harvest and plant height.

Table 1

Analysis of variance of morphological and yield characteristics in pepper crosses o

\begin{tabular}{|c|c|c|c|c|c|c|c|c|c|c|c|c|c|}
\hline S.o.V. & df & $\begin{array}{l}\text { Days to } \\
\text { seed } \\
\text { germination }\end{array}$ & $\begin{array}{l}\text { Days to } \\
\text { flowering }\end{array}$ & $\begin{array}{l}\text { days to } \\
\text { fruit set }\end{array}$ & $\begin{array}{l}\text { days to } \\
\text { fruit } \\
\text { harvest }\end{array}$ & $\begin{array}{l}\text { Fruit } \\
\text { length }\end{array}$ & $\begin{array}{l}\text { Fruit } \\
\text { width }\end{array}$ & $\begin{array}{l}\text { Pericarp } \\
\text { thickness }\end{array}$ & $\begin{array}{l}\text { Fruit } \\
\text { volume }\end{array}$ & Seed no. & $\begin{array}{l}\text { seed } \\
\text { fresh } \\
\text { weight }\end{array}$ & $\begin{array}{l}\text { seed } \\
\text { dry } \\
\text { weight }\end{array}$ & $\begin{array}{l}\text { lobes } \\
\text { no./fruit }\end{array}$ \\
\hline Hybrid & 20 & $9.64^{\star *}$ & $228.26^{\star \star}$ & $240.37^{\star \star}$ & $100.06^{\text {n.s }}$ & $41.17^{\star \star}$ & $10.61^{\star \star}$ & $0.26^{\star \star}$ & $241.22^{\star *}$ & $991.47^{\text {n.s }}$ & $0.18^{\mathrm{n} . \mathrm{s}}$ & $0.05^{\text {n.s }}$ & $0.23^{\text {** }}$ \\
\hline Error & 42 & 0.40 & 101.25 & 100.86 & 93.55 & 5.46 & 1.97 & 4.62 & 30.33 & 955.30 & 0.16 & 0.05 & 0.08 \\
\hline CV & & 0.63 & 11.50 & 9.84 & 5.81 & 14.52 & 2.35 & 0.08 & 3.59 & 35.92 & 28.30 & 49.77 & 8.78 \\
\hline
\end{tabular}

** $P<0.01$

* $\mathrm{P}<0.05$

n.s not significant 
Table 2

Means comparison analyzing using Duncan's multiple range test for morphological and yield charact

\begin{tabular}{|c|c|c|c|c|c|c|c|c|c|c|c|c|}
\hline $\begin{array}{l}\text { Crosses } \\
\text { (DH) }\end{array}$ & $\begin{array}{l}\text { Days to } \\
\text { seed } \\
\text { germination }\end{array}$ & $\begin{array}{l}\text { Days to } \\
\text { flowering }\end{array}$ & $\begin{array}{l}\text { days to } \\
\text { fruit set }\end{array}$ & $\begin{array}{l}\text { days to } \\
\text { fruit } \\
\text { harvest }\end{array}$ & $\begin{array}{l}\text { Fruit } \\
\text { length }\end{array}$ & $\begin{array}{l}\text { Fruit } \\
\text { width }\end{array}$ & $\begin{array}{l}\text { Pericarp } \\
\text { thickness }\end{array}$ & $\begin{array}{l}\text { Fruit } \\
\text { volume }\end{array}$ & Seed no. & $\begin{array}{l}\text { seed } \\
\text { fresh } \\
\text { weight }\end{array}$ & $\begin{array}{l}\text { seed } \\
\text { dry } \\
\text { weight }\end{array}$ & $\begin{array}{l}\text { lobe } \\
\text { no./ }\end{array}$ \\
\hline $55 * 64$ & $11.00 \mathrm{a}$ & 87.33a-d & $98.50 a-d$ & $169.01 a$ & 76.10ab & 57.77eg & 6.27a-d & $156.00 \mathrm{~cd}$ & 107.05ab & $1.58 \mathrm{abc}$ & $0.54 \mathrm{ab}$ & 3.87 \\
\hline $55 * 57$ & $11.00 \mathrm{a}$ & 86.17a-d & $96.17 \mathrm{abc}$ & $163.51 \mathrm{a}$ & $70.53 a-d$ & $59.29 \mathrm{~b}-\mathrm{e}$ & $6.40 \mathrm{abc}$ & $154.64 \mathrm{~cd}$ & 79.94ab & $1.16 \mathrm{abc}$ & $0.45 a b$ & 3.40 \\
\hline $55 * 53$ & $13.00 \mathrm{bc}$ & $81.00 \mathrm{abc}$ & $87.83 a$ & $161.03 a$ & $73.08 a-d$ & 61.93abc & $6.13 \mathrm{a}-\mathrm{d}$ & $173.47 a$ & 109.93ab & $1.62 \mathrm{abc}$ & $0.59 a b$ & 3.40 \\
\hline $55 * 217$ & $12.00 \mathrm{ab}$ & $84.83 a-d$ & $95.17 a b$ & $159.56 a$ & $68.43 \mathrm{cde}$ & $60.78 \mathrm{bcd}$ & 6.37a-d & $150.07 d$ & $94.65 a b$ & $1.63 \mathrm{abc}$ & $0.59 a b$ & 3.39 \\
\hline $55 * 90$ & $16.00 \mathrm{e}$ & 89.00a-e & $\mathrm{e}^{112.17 b-}$ & $173.77 a$ & $69.53 \mathrm{bcd}$ & 57.92eg & 6.27a-d & 138.80ef & $52.45 b$ & $0.94 c$ & $0.26 a b$ & 3.35 \\
\hline $55 * 218$ & $16.00 \mathrm{e}$ & 89.83a-e & $e^{100.33 a-}$ & $172.77 a$ & $69.78 \mathrm{bcd}$ & $59.46 \mathrm{~b}-\mathrm{e}$ & 6.35a-d & 146.78de & 88.73ab & 1.37abc & $0.49 a b$ & 3.30 \\
\hline $64 * 57$ & $16.00 \mathrm{e}$ & 92.00a-e & $e^{105.67 a-}$ & $158.61 a$ & $77.55 a$ & $57.02 \mathrm{~g}$ & 6.37a-d & $162.55 \mathrm{bc}$ & 71.22ab & $1.22 \mathrm{abc}$ & $0.38 a b$ & 3.29 \\
\hline $64 * 53$ & $15.00 \mathrm{de}$ & $107.83 \mathrm{e}$ & $118.83 \mathrm{e}$ & $169.08 a$ & $74.98 \mathrm{abc}$ & $57.22 \mathrm{~g}$ & $5.84 d$ & $12.96 \mathrm{~cd}$ & 78.27ab & 1.16abc & $0.30 \mathrm{ab}$ & 3.23 \\
\hline $64 * 217$ & $16.00 \mathrm{e}$ & $103.00 \mathrm{de}$ & $\mathrm{e}^{109.83 \mathrm{~b}-}$ & $172.73 a$ & $70.95 a-d$ & $60.91 a-d$ & $5.93 \mathrm{~cd}$ & 166.23ab & $124.50 a$ & $1.80 \mathrm{ab}$ & $0.60 \mathrm{ab}$ & 3.20 \\
\hline $64 * 90$ & $13.00 \mathrm{bc}$ & $99.67 \mathrm{~b}-\mathrm{e}$ & $116.50 \mathrm{de}$ & $173.14 a$ & $72.92 a-d$ & $56.20 \mathrm{~g}$ & $5.93 \mathrm{~cd}$ & $145.86 \mathrm{de}$ & $72.25 a b$ & 1.19abc & $0.19 b$ & 3.19 \\
\hline $64 * 218$ & $14.00 \mathrm{~cd}$ & 81.17abc & $88.50 a$ & $159.53 a$ & 69.71bcd & $57.92 \mathrm{eg}$ & $6.18 \mathrm{a}-\mathrm{d}$ & $150.367 d$ & 87.86ab & $1.35 \mathrm{abc}$ & $0.48 a b$ & 3.18 \\
\hline $57 * 53$ & $13.00 \mathrm{bc}$ & 85.17a-d & $e^{100.33 a-}$ & 159.73a & $61.73 e$ & $60.25 b-e$ & $5.32 e$ & $134.89 f$ & $72.46 a b$ & $1.27 \mathrm{abc}$ & $0.34 a b$ & 3.11 \\
\hline $57 * 217$ & $13.00 \mathrm{bc}$ & $81.83 a b c$ & 97.33a-d & $164.98 a$ & $60.50 \mathrm{bcd}$ & $63.51 a$ & $6.07 \mathrm{bcd}$ & 167.59ab & $100.96 a b$ & 1.54abc & $0.54 a b$ & 3.06 \\
\hline $57 * 90$ & $15.00 \mathrm{de}$ & $100.83 \mathrm{cde}$ & $\mathrm{e}^{115.50 \mathrm{c}-}$ & $160.53 a$ & $68.46 \mathrm{cde}$ & $60.00 \mathrm{~b}-\mathrm{e}$ & $6.26 a-d$ & $155.80 \mathrm{~cd}$ & 87.07ab & $1.29 \mathrm{abc}$ & $0.50 \mathrm{ab}$ & 2.99 \\
\hline $57 * 218$ & $12.00 \mathrm{ab}$ & 79.83ab & $98.67 a-d$ & $165.92 a$ & 66.13de & 61.12a-d & 6.35a-d & $152.38 \mathrm{~cd}$ & 105.73ab & $1.68 a b$ & $0.63 a b$ & 2.99 \\
\hline $53 * 217$ & $12.00 \mathrm{ab}$ & $80.17 a b$ & $e^{100.83 a-}$ & $169.23 a$ & $67.76 \mathrm{cde}$ & $60.91 a-d$ & 6.34a-d & $155.43 \mathrm{~cd}$ & $94.62 a b$ & $1.53 \mathrm{abc}$ & $0.52 \mathrm{ab}$ & 2.96 \\
\hline $53 * 90$ & $15.33 e$ & $88.00 a-d$ & $\begin{array}{l}\mathrm{e}^{110.00 \mathrm{~b}-} \\
\end{array}$ & 174.19a & 69.76bcd & $58.74 \mathrm{deg}$ & $5.87 \mathrm{~cd}$ & 147.94de & $70.50 a b$ & $1.09 \mathrm{bc}$ & $0.32 \mathrm{ab}$ & 2.92 \\
\hline $53 * 218$ & $12.00 \mathrm{ab}$ & $77.17 a$ & 93.83ab & $167.18 \mathrm{a}$ & $65.91 \mathrm{de}$ & 61.17a-d & 6.35a-d & $154.15 \mathrm{~cd}$ & $70.24 a b$ & 1.31abc & $0.39 a b$ & 2.87 \\
\hline $217 * 90$ & $11.00 \mathrm{a}$ & $77.50 \mathrm{a}$ & 95.83abc & $158.99 a$ & $66.00 \mathrm{de}$ & 61.97ab & $6.28 a-d$ & $149.46 d$ & 95.48ab & $1.52 \mathrm{abc}$ & $0.57 a b$ & 2.83 \\
\hline $217 * 218$ & $14.00 \mathrm{~cd}$ & $84.83 a-d$ & $\begin{array}{l}\text { 104.00a- } \\
\text { el }\end{array}$ & $174.17 a$ & $70.63 a-d$ & $59.20 \mathrm{cde}$ & $6.54 \mathrm{ab}$ & $150.50 d$ & 107.94ab & $1.82 a$ & $0.64 a b$ & 2.75 \\
\hline $90 * 218$ & $12.00 \mathrm{ab}$ & 79.83ab & 98.83a-d & $170.32 a$ & 66.87cde & $60.41 c-e$ & $6.67 a$ & $155.71 \mathrm{~cd}$ & 69.20ab & $1.21 \mathrm{abc}$ & $0.33 a b$ & 2.64 \\
\hline
\end{tabular}

Within a column, means followed by similar letters are not significantly different according to DMRT at $a=0.05$

\section{General and specific combining ability analysis}

The results of analysis of variance for the studied traits showed a significant effect of genotype in all traits, except number of days to harvesting, seed number, dry seed weight, main stem dimeter and plant height (Table 3). According to Table 3, due to the significant effect of genotype on the most of the evaluated traits, diallel analysis for genetic analysis and estimation of genetic characteristics for these traits was possible. The effect of general and specific combining ability was significant for all traits except fruit dry weight, number of seeds, fruit fresh weight, seed dry weight, number of lobes per fruit and main stem diameter (Table 3). 
Table 3

Analysis of Variance of diallel cross design for morphological and yield characteristics in

\begin{tabular}{|c|c|c|c|c|c|c|c|c|c|c|c|c|c|}
\hline s.o.v. & $d f$ & $\begin{array}{l}\text { Days to } \\
\text { seed } \\
\text { germination }\end{array}$ & $\begin{array}{l}\text { Days to } \\
\text { fruit set }\end{array}$ & $\begin{array}{l}\text { Days to } \\
\text { flowering }\end{array}$ & $\begin{array}{l}\text { Days to } \\
\text { fruit } \\
\text { harvesting }\end{array}$ & $\begin{array}{l}\text { Fruit } \\
\text { length }\end{array}$ & $\begin{array}{l}\text { Fruit } \\
\text { width }\end{array}$ & $\begin{array}{l}\text { Pericarp } \\
\text { thickness }\end{array}$ & $\begin{array}{l}\text { Fruit } \\
\text { fresh } \\
\text { weight }\end{array}$ & $\begin{array}{l}\text { Fruit } \\
\text { dry } \\
\text { weight }\end{array}$ & Seed no. & $\begin{array}{l}\text { Seed } \\
\text { fresh } \\
\text { weight }\end{array}$ & $\begin{array}{l}S \\
d \\
n\end{array}$ \\
\hline Replication & 2 & $0.12^{n \cdot s}$ & $393.90^{*}$ & $116.96^{\text {n.s }}$ & $284.93^{*}$ & $10.43^{n \cdot s}$ & $2.55^{\mathrm{n} . \mathrm{s}}$ & $0.18^{n . s}$ & $49.21^{\mathrm{n} . \mathrm{s}}$ & $1.13^{\mathrm{n} . \mathrm{s}}$ & $1899.24^{\text {n.s }}$ & $0.49^{\star}$ & 0 \\
\hline Entry & 20 & $9.28^{\star \star}$ & $228.63^{\star \star}$ & $236.90^{\star \star}$ & $92.94^{\text {n.s }}$ & $41.44^{\star \star}$ & $10.61^{\star \star}$ & $0.26^{* \star}$ & $118.88^{\star *}$ & $1.46^{* \star}$ & $1109.01^{\text {n.s }}$ & $0.22^{*}$ & 0 \\
\hline GCA & 6 & $3.24^{\star *}$ & $350.22^{* *}$ & $343.82^{*}$ & $93.41^{\star *}$ & $83.21^{\star *}$ & $23.08^{\star *}$ & $0.45^{* \star}$ & $125.66^{* *}$ & $0.73^{n . s}$ & $1600.08^{\text {n.s }}$ & $0.38^{\mathrm{n} . \mathrm{s}}$ & 0 \\
\hline SCA & 14 & $12.39^{* *}$ & $175.99^{*}$ & $196.03^{* \star}$ & $102.90^{\star \star}$ & $23.16^{* *}$ & $5.27^{\star \star}$ & $0.18^{*}$ & $116.25^{\star \star}$ & $1.85^{n . s}$ & $678.96^{\text {n.s }}$ & $0.09^{n . s}$ & 0 \\
\hline Error & 40 & 0.58 & 85.33 & 101.10 & 87.37 & 14.66 & 1.94 & 0.07 & 48.81 & 0.54 & 832.82 & 0.11 & 0 \\
\hline $\mathrm{cV}$ & & 5.66 & 10.56 & 9.85 & 5.61 & 5.48 & 2.33 & 4.41 & 8.01 & 9.84 & 32.92 & 23.41 & 4 \\
\hline
\end{tabular}

** $P<0.01$

* $\mathrm{P}<0.05$

n.s not significant

The specific combining ability (SCA) of the all hybrids (cross combinations) investigated from diallel cross of seven doubled haploid lines of pepper was shown in Table 4. The diallel cross of DH55×DH64 indicated the highest positive SCA value for average yield per plant, however it showed the lowest SCA effect for number of days to germination (Table 4). The highest SCA values of number of days to flowering and fruit set were obtained in hybrid combination DH64×DH53 (Table 4). The cross combination DH55×DH53 demonstrated high positive SCA values for fruit width, fruit fresh weight, fruit dry weight and fruit volume. Meanwhile, highly significant SCA effects for fruit length and pericarp thickness were proved by hybrid DH64×DH57 (Table 4). In addition, the SCA effects were positively significant for fruit number per plant, lobes number per fruit and main stem length in hybrid DH57×DH90 (Table 4). 
Table 4

Estimation of specific combining ability (SCA) for different traits of evaluated pepper hybrids using diallel cross design

\begin{tabular}{|c|c|c|c|c|c|c|c|c|c|c|c|c|c|}
\hline Hybrids & $\begin{array}{l}\text { Days to } \\
\text { seed } \\
\text { germination }\end{array}$ & $\begin{array}{l}\text { Days to } \\
\text { flowering }\end{array}$ & $\begin{array}{l}\text { Days to } \\
\text { fruit set }\end{array}$ & $\begin{array}{l}\text { Fruit } \\
\text { length }\end{array}$ & $\begin{array}{l}\text { Fruit } \\
\text { width }\end{array}$ & $\begin{array}{l}\text { Pericarp } \\
\text { thickness }\end{array}$ & $\begin{array}{l}\text { Fruit } \\
\text { fresh } \\
\text { weight }\end{array}$ & $\begin{array}{l}\text { Fruit } \\
\text { dry } \\
\text { weight }\end{array}$ & $\begin{array}{l}\text { Seed } \\
\text { fresh } \\
\text { weight }\end{array}$ & $\begin{array}{l}\text { Fruit } \\
\text { no./plant }\end{array}$ & $\begin{array}{l}\text { Lobes } \\
\text { no./fruit }\end{array}$ & $\begin{array}{l}\text { Fruit } \\
\text { density }\end{array}$ & $\begin{array}{l}\text { Ma } \\
\text { stel } \\
\text { len! }\end{array}$ \\
\hline $55 * 64$ & $-2.98^{\star *}$ & $-8.03^{n . s}$ & $-3.90^{\mathrm{n} . \mathrm{s}}$ & $-0.08^{n . s}$ & $0.52^{n . s}$ & $0.08^{\text {n.s }}$ & $1.99^{n . s}$ & $0.7^{*}$ & $0.21^{\mathrm{n} . \mathrm{s}}$ & $2.16^{\mathrm{n} . \mathrm{s}}$ & $0.16^{\text {n.s }}$ & $0.02^{n . s}$ & -0.8 \\
\hline $55 * 57$ & $-1.98^{\star *}$ & $-0.17^{n . s}$ & $-1.40^{\mathrm{n} . \mathrm{s}}$ & $0.01^{\mathrm{n} . \mathrm{s}}$ & $-0.80^{\text {n.s }}$ & $0.16^{\mathrm{n} . \mathrm{s}}$ & $-0.27^{n . s}$ & $-0.16^{n \cdot s}$ & $-0.18^{n . s}$ & $1.16^{\text {n.s }}$ & $-0.13^{n \cdot s}$ & $-0.11^{\mathrm{n} . \mathrm{s}}$ & -0.5 \\
\hline $55 * 53$ & $-0.04^{\text {n.s }}$ & $-4.03^{n . s}$ & $-10.67^{*}$ & $2.70^{\text {n.s }}$ & $2.04^{\star *}$ & $0.08^{\text {n.s }}$ & $14.72^{\star *}$ & $1.56^{* *}$ & $0.32^{\text {n.s }}$ & $0.22^{\mathrm{n} . \mathrm{s}}$ & $0.30^{*}$ & $20.52^{\star \star}$ & 2.81 \\
\hline $55 * 217$ & $-0.58^{n . s}$ & $1.23^{\mathrm{n} . \mathrm{s}}$ & $-0.27^{\mathrm{n} . \mathrm{s}}$ & $-1.97^{n . s}$ & $-0.53^{n . s}$ & $-0.02^{n \cdot s}$ & $-7.02^{*}$ & $-1.02^{\star \star}$ & $-0.04^{\text {n.s }}$ & $-0.84^{\mathrm{n} . \mathrm{s}}$ & $-0.21^{\mathrm{n} . \mathrm{s}}$ & $-6.97^{\star *}$ & $0.3^{\circ}$ \\
\hline $55 * 90$ & $2.56^{* *}$ & $0.87^{n \cdot s}$ & $7.57^{n \cdot s}$ & $-0.91^{\mathrm{n} . \mathrm{s}}$ & $-0.97^{\text {n.s }}$ & $-0.07^{\text {n.s }}$ & $-5.32^{\mathrm{n} . \mathrm{s}}$ & $-0.67^{n \cdot s}$ & $-0.22^{\text {n.s }}$ & $-0.64^{\mathrm{n} . \mathrm{s}}$ & $-0.10^{\text {n.s }}$ & $-9.09^{* *}$ & -3.7 \\
\hline $55 * 218$ & $3.02^{\star \star}$ & $10.13^{*}$ & $8.67^{n . s}$ & $0.24^{\mathrm{n} . \mathrm{s}}$ & $-0.25^{\text {n.s }}$ & $-0.22^{\text {n.s }}$ & $-4.09^{n . s}$ & $-0.43^{n \cdot s}$ & $-0.09^{\text {n.s }}$ & $-2.04^{\mathrm{n} . \mathrm{s}}$ & $0.05^{\mathrm{n} . \mathrm{s}}$ & $-4.37^{\text {n.s }}$ & 1.91 \\
\hline $64 * 57$ & $1.82^{\star *}$ & $-4.90^{n \cdot s}$ & $-1.77^{n . s}$ & $4.08^{*}$ & $-1.05^{\mathrm{n} . \mathrm{s}}$ & $0.39^{\star *}$ & $-0.23^{n . s}$ & $0.59^{n . s}$ & $-0.12^{\text {n.s }}$ & $1.56^{\mathrm{n} . \mathrm{s}}$ & $-0.28^{*}$ & $4.96^{\mathrm{n} . \mathrm{s}}$ & -4.3 \\
\hline $64 * 53$ & $0.76^{\star \star}$ & $12.23^{\star \star}$ & $12.13^{\star *}$ & $1.65^{\mathrm{n} . \mathrm{s}}$ & $-0.65^{\text {n.s }}$ & $0.04^{\mathrm{n} . \mathrm{s}}$ & $-2.40^{\mathrm{n} . \mathrm{s}}$ & $-0.95^{\star \star}$ & $-0.15^{\mathrm{n} . \mathrm{s}}$ & $-1.38^{n \cdot s}$ & $-0.29^{*}$ & $-2.83^{n \cdot s}$ & $3.4^{\prime}$ \\
\hline $64 * 217$ & $2.22^{\star *}$ & $8.83^{n . s}$ & $4.53^{n . s}$ & $-2.39^{n . s}$ & $1.62^{*}$ & $-0.21^{\mathrm{n} . \mathrm{s}}$ & $3.94^{\mathrm{n} . \mathrm{s}}$ & $-0.22^{n \cdot s}$ & $0.13^{\mathrm{n} . \mathrm{s}}$ & $-0.44^{\mathrm{n} . \mathrm{s}}$ & $0.27^{*}$ & $6.36^{*}$ & $2.0^{\prime}$ \\
\hline $64 * 90$ & $-1.64^{\star *}$ & $0.97^{\text {n.s }}$ & $2.03^{n \cdot s}$ & $-0.48^{n . s}$ & $-0.67^{n . s}$ & $-0.16^{\text {n.s }}$ & $-0.61^{\mathrm{n} . \mathrm{s}}$ & $-0.40^{\text {n.s }}$ & $0.04^{\mathrm{n} . \mathrm{s}}$ & $-1.58^{n . s}$ & $0.09^{n . s}$ & $-4.87^{n . s}$ & $3.9 i$ \\
\hline $64 * 218$ & $-0.18^{n \cdot s}$ & $-9.10^{\text {n.s }}$ & $-13.03^{\star \star}$ & $-2.78^{n . s}$ & $0.23^{n . s}$ & $-0.14^{\mathrm{n} . \mathrm{s}}$ & $-2.67^{n . s}$ & $0.27^{n . s}$ & $-0.10^{\text {n.s }}$ & $-0.31^{\mathrm{n} . \mathrm{s}}$ & $-0.03^{n . s}$ & $-3.63^{n \cdot s}$ & -4.3 \\
\hline $57 * 53$ & $-0.24^{\mathrm{n} . \mathrm{s}}$ & $-1.40^{\text {n.s }}$ & $-1.53^{n . s}$ & $-5.94^{\star \star}$ & $-0.45^{\mathrm{n} . \mathrm{s}}$ & $-0.53^{\star *}$ & $-9.54^{\star \star}$ & $-1.00^{\star *}$ & $-0.01^{n . s}$ & $-0.71^{\mathrm{n} . \mathrm{s}}$ & $0.07^{\text {n.s }}$ & $-19.68^{\star *}$ & -1.7 \\
\hline $57 * 217$ & $0.22^{\mathrm{n} . \mathrm{s}}$ & $-3.30^{\text {n.s }}$ & $-3.13^{\mathrm{n} . \mathrm{s}}$ & $1.82^{\mathrm{n} . \mathrm{s}}$ & $1.40^{*}$ & $-0.12^{n \cdot s}$ & $5.96^{\text {n.s }}$ & $0.50^{\mathrm{n} . \mathrm{s}}$ & $-0.11^{\mathrm{n} . \mathrm{s}}$ & $-0.11^{\mathrm{n} . \mathrm{s}}$ & $-0.09^{n . s}$ & $8.93^{\star \star}$ & -2.8 \\
\hline $57 * 90$ & $1.36^{\star *}$ & $11.17^{\star}$ & $5.86^{\mathrm{n} . \mathrm{s}}$ & $0.72^{\text {n.s }}$ & $0.30^{\text {n.s }}$ & $0.12^{\mathrm{n} \cdot \mathrm{s}}$ & $2.95^{\mathrm{n} . \mathrm{s}}$ & $0.55^{\mathrm{n} . \mathrm{s}}$ & $0.16^{\mathrm{n} . \mathrm{s}}$ & $-2.58^{\star}$ & $0.37^{\star \star}$ & $6.29^{*}$ & 6.3: \\
\hline $57 * 218$ & $-1.18^{\star \star}$ & $-1.40^{\text {n.s }}$ & $1.97^{\text {n.s }}$ & $-0.70^{\text {n.s }}$ & $0.61^{\text {n.s }}$ & $-0.02^{\text {n.s }}$ & $1.13^{\mathrm{n} . \mathrm{s}}$ & $-0.47^{n \cdot s}$ & $0.25^{\mathrm{n} . \mathrm{s}}$ & $0.69^{\text {n.s }}$ & $0.06^{\mathrm{n} . \mathrm{s}}$ & $-0.40^{\text {n.s }}$ & 3.21 \\
\hline $53 * 217$ & $-0.84^{\star *}$ & $-3.67^{n . s}$ & $1.10^{\text {n.s }}$ & $0.22^{n . s}$ & $-1.01^{\mathrm{n} . \mathrm{s}}$ & $0.33^{*}$ & $0.07^{n . s}$ & $0.55^{n . s}$ & $-0.08^{n . s}$ & $0.62^{n . s}$ & $0.11^{\mathrm{n} . \mathrm{s}}$ & $-1.42^{\text {n.s }}$ & -0.9 \\
\hline $53 * 90$ & $1.62^{\star \star}$ & $-0.37^{\text {n.s }}$ & $1.10^{\mathrm{n} . \mathrm{s}}$ & $2.16^{\mathrm{n} . \mathrm{s}}$ & $-0.77^{\text {n.s }}$ & $-0.09^{n . s}$ & $-2.30^{n \cdot s}$ & $-0.16^{\mathrm{n} \cdot \mathrm{s}}$ & $0.00^{\text {n.s }}$ & $2.16^{\mathrm{n} . \mathrm{s}}$ & $-0.27^{\star}$ & $0.23^{n . s}$ & -1.0 \\
\hline $53 * 218$ & $-1.24^{\star *}$ & $-2.77^{n . s}$ & $-2.13^{n \cdot s}$ & $-0.79^{n . s}$ & $0.85^{n . s}$ & $0.16^{\mathrm{n} . \mathrm{s}}$ & $-0.53^{n . s}$ & $0.01^{\mathrm{n} . \mathrm{s}}$ & $-0.08^{\text {n.s }}$ & $-0.91^{\mathrm{n} . \mathrm{s}}$ & $0.07^{n \cdot s}$ & $3.17^{n . s}$ & -2.5 \\
\hline $217 * 90$ & $-0.89^{\star \star}$ & $1.73^{\text {n.s }}$ & $-5.80^{\mathrm{n} . \mathrm{s}}$ & $-0.89^{n . s}$ & $1.34^{\mathrm{n} . \mathrm{s}}$ & $0.12^{\mathrm{n} . \mathrm{s}}$ & $1.03^{n . s}$ & $0.68^{\mathrm{n} . \mathrm{s}}$ & $0.2^{\text {n.s }}$ & $-2.16^{\mathrm{n} . \mathrm{s}}$ & $0.36^{*}$ & $3.95^{n . s}$ & $3.4^{\circ}$ \\
\hline $217 * 218$ & $1.22^{\star \star}$ & $6.33^{n . s}$ & $9.43^{*}$ & $3.93^{\star}$ & $-2.53^{* *}$ & $0.02^{\text {n.s }}$ & $-1.03^{n . s}$ & $0.06^{\mathrm{n} . \mathrm{s}}$ & $0.06^{\mathrm{n} . \mathrm{s}}$ & $0.36^{\text {n.s }}$ & $-0.08^{n . s}$ & $-4.56^{n . s}$ & $4.2 i$ \\
\hline $90 * 218$ & $-1.64^{\star \star}$ & $-3.20^{n . s}$ & $-4.90^{\mathrm{n} . \mathrm{s}}$ & $0.11^{\mathrm{n} . \mathrm{s}}$ & $1.08^{n . s}$ & $0.19^{n . s}$ & $7.20^{\star}$ & $0.56^{\mathrm{n} . \mathrm{s}}$ & $-0.03^{n . s}$ & $2.22^{\mathrm{n} . \mathrm{s}}$ & $-0.07^{n . s}$ & $9.79^{\star *}$ & -2.6 \\
\hline $\operatorname{SE}\left(g_{i}\right)$ & 0.34 & 14.19 & 14.13 & 3.26 & 0.90 & 0.02 & 5.66 & 0.05 & 0.01 & 0.87 & 0.02 & 9.01 & 3.21 \\
\hline
\end{tabular}

\section{$* * \mathrm{P}<0.01$}

$* \mathrm{P}<0.05$

\section{n.s.: not significant}

In general, estimation of genetic variance components, narrow-sense heritability and mean squared ratio of GCA to SCA indicated that gene action in all gene loci for fruit width and length and seed fresh weight were positive (Table 5). 
Table 5

Estimation of mean squared ratio of general to specific combining ability, type of gene action, additive and dominance variances, narrow-sense heritability and the best combination(hybrid) for different traits in doubled haploid lines of pepper using diallel cross design.

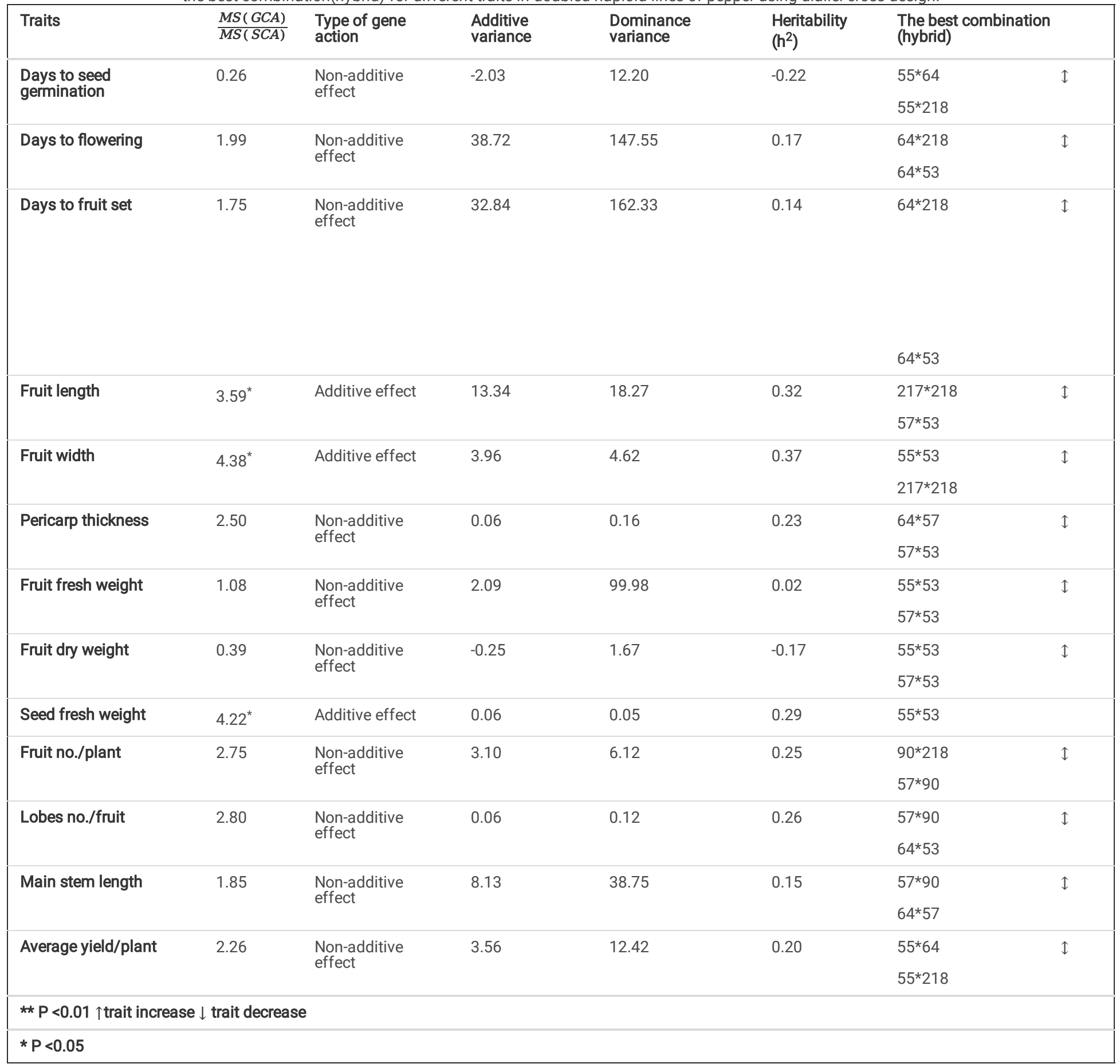

The ratio of $\frac{M S(G C A)}{M S(S C A)}$ was not found to be significant in all traits except fruit length, fruit width and seed fresh weight, which indicated the relative importance of non-additive effects of genes (dominance effect) and low amount of additive effects in controlling these traits (Table 5). Therefore, the significance of the fruit length, fruit width and seed fresh weight indicated the additive gene actions on the inheritance of the above traits (Table 5).

The low amount of narrow-sense heritability for average yield per plant (0.20) also emphasizes the control of this trait under the influence of non-additive effects of genes. In addition, the amount of dominance variance was more than additive variance and therefore the dominance effect was greater than the additive effect in controlling the average yield per plant (Table 5). Based on the results, selection for this trait cannot be successful and attention to hybridization can be very useful.

\section{Discussion}

Securing the higher yield plays as essential role in improvement of sweet pepper cultivars. In this study, the significant differences were founded for most evaluated characters such as fruit length, fruit width, fruit thickness and fruit volume, fruit fresh and dry weight, fruit number per plant, lobes number per fruit and average yield per plant. Significant differences between bell pepper or hot pepper genotypes were also observed by other researchers (Naresh et al., 2016; 
Devi et al., 2018; Aiswarya et al., 2020). Naresh et al. (2016) reported the significant differences for all traits according to ANOVA analysis in chilli pepper. In addition, analysis of variance showed the significant differences in bell pepper for all traits by Devi et al. (2018).

The parents with good performance may not always be able to pass on their superior traits to the hybrids or the next generation, hence, combining ability studies are required (Kadambavanasundaram, 1980). Combining ability analysis manifested high and significant effects of GCA and SCA for the most studied characters. General combining ability (GCA) is an average performance of a line in the crosses that is controlled by additive gene action and is fixable, although specific combining ability (SCA) is the parental lines performance in a specific cross combination, that is non fixable due to non-additive gene action (Sprague and Tatum, 1942; Rukundo et al., 2017). Furthermore, the low narrow-sense heritability indicates a lack of relative influence of selection on superior offspring production, and therefore requires large populations, offspring test and complicated statistical methods to identify suitable genotypes (Taleei and Noormohamadi, 1994). We founded significant differences in the GCA and SCA variances for most studied traits that demonstrates the effect of both additive and non-additive gene actions in hybrid performance. In addition, according to Table 5, heritability was small for most of traits such as average yield per plant, fruit fresh weight and fruit dry weight, which have also been reported by other researchers (Hasanuzzaman et al., 2012; Bhutia et al., 2015; Aiswarya et al., 2020). The significant SCA effects was reported by Das et al., (2020) in five crosses for fruit yield per plant, PDI of downy mildew, total sugar content, number of fruit per plant, fruit diameter, days to first female flower appearance, and vine length.

High dominance variance compared to additive variance for days to flowering, days to fruit set, fruit fresh weight, fruit length and fruit width provides an appropriate genetic potential for heterosis breeding in sweet pepper because, the optimal use of heterosis requires the dominance effects (Falconer and Mackay, 1996). The high heterotic effect over standard parent was reported by Nimitha et al., (2018) for fruit yield per plant and number of fruits per plant in cucumber that can be used in commercial cultivation. Manggoel et al., (2021) reported the significant diversity between the cucumber genotypes studied. The findings from this study showed the priority of dominant gene effects over additive gene effects for the genetic control of vine length and number of leaves per plant (Manggoel et al., 2021).

In opposite, high additive gene action was obtained for the mean fruit mass and fruit length of sweet pepper (Yadahalli et al., 2017; Chagas et al., 2019).

\section{Conclusions}

In this research, general and specific combining ability of seven desired DH lines of sweet pepper were investigated. The lines were created using microspore culture and examined by the top-cross method. The highest positive GCA value for average yield per plant was observed in DH55 line and then belonged to DH64, DH57, DH53, DH217, DH90 and DH218, respectively. These cross combinations can be studied for improvement of superior entries and applying hybrid vigor in sweet pepper. Based on specific combining ability representative, $64 \times 57$ cross combination was the best cross for creating $F_{1}$ hybrid with the highest fruit length. In addition, the best combination (hybrid) for average yield per plant was $55 \times 64$ cross, while this combination for fruit fresh weight, fruit dry weight, fruit width and seed fresh weight was related to $55 \times 53$ cross. Therefore, this study founded that the above promising crosses can be designated for exploiting commercial hybrids or applying in breeding programs of sweet pepper.

\section{Abbreviations}

ANOVA: Analysis of variance; DH: Doubled haploid; GCA: General combining ability; SCA: Specific combining ability; CRD: Completely randomized design, df: degree of freedom.

\section{Declarations}

\section{Acknowledgements}

The authors gratefully thank the Agricultural Biotechnology Research Institute of Iran, for granting a Project No. 04-05-05-004-950050.

\section{Author contribution statement}

A Z B performed diallel crosses and data analyses of the experiment, M E.S (corresponding author) contributed in the supervising of the whole process and correcting the manuscript, $\mathrm{KM}$ and S M M supervised the project and contributed in statistical analyses.

\section{Compliance with ethical standards}

There is not any ethical standard related to the present research article.

\section{Conflict of interest}

The authors declare that they have no conflict of interest.

\section{References}

1. Aiswarya, C.S., Vijeth, S., Sreelathakumary, I., Kaushik, P. 2020. Diallel analysis of chilli pepper (Capsicum annuum L.) genotypes for morphological and fruit biochemical traits. Plants. 9(1): 1-15. https://doi.org/10.3390/plants9010001.

2. Bhutia, N.D., Seth, T., Shende, V.D., Dutta, S., Chattopadhyay, A. 2015. Estimation of Heterosis, dominance effect and genetic control of fresh fruit yield, quality and leaf curl disease severity traits of chilli pepper (Capsicum annuum L.). Scientia Horticulturae. 182: 47-55. [CrossRef] 
3. Boswell, V.R. 1937. Genetics of tomatoes, peppers, and eggplant. In: Yearbook of agriculture. Washington D.C., U.S. Department of agriculture. $176-206$.

4. Chagas, J.T.B., Santos, P.R., Daher, R., Stida, W.F., Vidal, A.K., Nascimento, M.R., Costa, K.D.S., Vivas, M., Júnior, A.T.A., Menezes, D. 2019. Estimation of genetic merit of diallel hybrids of sweet pepper by mixed models. Ciência Rural. 49(8): 1-8. https://doi.org/10.1590/0103-8478cr20180968.

5. Çömlekçioğlu, N., Ellialtıoğlu, Ş.Ş. 2018. Review on the Research carried out on in vitro Androgenesis of Peppers (Capsicum annuum L.) in Turkey. Review Paper: Research Journal of Biotechnology. 13(6): 75- 84.

6. Das, S.P., Mandal, A.R., Maurya, P.K., Bhattacharjee, T., Banerjee, S., Mandal, A.S., Chattopadhyay, A. 2020. Genetic control of economic traits and evidence of economic heterosis in crosses involving monoecious cucumber genotypes. International Journal of Vegetable Science. 1-22. DOI: 10.1080/19315260.2019.1639873.

7. Devi, M.B., Pathania, N.K., Thakur, N. 2018. Estimation of genetic variability, GCA and SCA effects for development of early and high yielding bell pepper hybrids suitable for protected cultivation. Journal of Applied and Natural Science, 10(1): 410-416. https://doi.org/10.31018/jans.v10i1.1639.

8. Dezfouli, P.M., Sedghi, M., Shariatpanahi, M.E., Niazian, M., Alizadeh, B. 2019. Assessment of general and specific combining abilities in doubled haploid lines of rapeseed (Brassica napus L.). Industrial Crops and Products. 141: 111754.

9. Esin, A.R.I., Yildirim, T., Mutlu, N., Büyükalaca, S., Gökmen, Ü., Akman, E. 2016. Comparison of different androgenesis protocols for doubled haploid plant production in ornamental pepper (Capsicum annuum L.). Turkish Journal of Biology. 40: 944-954. doi:10.3906/biy-1509-36.

10. Falconer, D.S., Mackay, T.F.C., 1996. Introduction to Quantitative Genetics. Prentice Hall.

11. Gil-Humanes, J., Barro, F. 2009. Production of Doubled Haploids in Brassica. In: Touraev, A., Forster, B.P., Jain, S.M. (eds) Advances in Haploid Production in Higher Plants. Springer, Dordrecht. https://doi.org/10.1007/978-1-4020-8854-4_4.

12. Griffing, B.R.U.C.E. 1956. Concept of general and specific combining ability in relation to diallel crossing systems. Aust. J. Biol. Sci. 9(4): $463-493$.

13. Hasanuzzaman, M., Hakim, M.A., Fersdous, J., Islam, M.M., Rahman, L. 2012. Combining ability and heritability analysis for yield and yield contributing characters in chilli (Capsicum annuum) landraces. Plant Omics. 5(4): 337-344.

14. Heidari-Zefreh, A.A., Shariatpanahi, M.E., Mousavi, A., Kalatejari, S. 2018. Enhancement of microspore embryogenesis induction and plantlet regeneration of sweet pepper (Capsicum annuum L.) using putrescine and ascorbic acid. Protoplasma. 256: 13-24. https://doi.org/10.1007/s00709-018-1268-3.

15. Jindal, S.K., Dhaliwal, M.S., Meena, O.M. 2019. Molecular advancements in male sterility systems of Capsicum: A review. Plant breeding. $139(1)$ : $42-64$. https://doi.org/10.1111/pbr.12757.

16. Kadambavanasundaram, M. 1980. Heterotic system in cultivated species of Gossypium. An appraisal (Abst). Genetic and crop improvement of heterotic systems. Pre-congress scientific meeting of XV International Congress of Genetics, TNAU, Coimbatore, pp. 20.

17. Kaushik, P., 2019a. Genetic Analysis for Fruit Phenolics Content, Flesh Color, and Browning Related Traits in Eggplant (Solanum melongena). Int. J. Mol. Sci. 20, 2990.

18. Kaushik, P., 2019b. Line $\times$ Tester Analysis for Morphological and Fruit Biochemical Traits in Eggplant (Solanum melongena L.) Using Wild Relatives as Testers. Agronomy. 9, 185.

19. Kaushik, P., Dhaliwal, M.S., 2018. Diallel Analysis for Morphological and Biochemical Traits in Tomato Cultivated under the Influence of Tomato Leaf Curl Virus. Agronomy. 8, 153.

20. Manggoel, W., Uguru, M.I., Ogbonna, P.E., Dasbak, M.A. 2021. Exploiting combining ability in a diallel cross between native and elite cucumber (Cucumis sativus L.) varieties. Journal of Plant Breeding and Crop Science. 13(3): 136-143. DOI: 10.5897/JPBCS2021.0955.

21. Möhring, J., Melchinger, A.E., Piepho, H.P. 2011. REML-based diallel analysis. Crop Sci. 51 (2): 470-478.

22. Naresh, P., Rao, V.K., Lavanya Reddy, B., Anand Reddy, C., Venkatachalapathi, V., Madhavi Reddy, K. 2016. Genetic analysis for fruit biochemical traits (capsaicinoids and carotenoids) and dry fruit yield in chilli (Capsicum annuum L.). Industrial Crops and Products. 94: 920-931. [CrossRef]

23. Nimitha, K., Acharya, R.R., Parmar, D.J. 2018. Exploitation of hybrid vigour through diallel analysis in cucumber (Cucumis sativus L.). Electronic Journal of Plant Breeding. 9(1): 60-65.

24. Rêgo, E.R., Rêgo M.M. 2016. Genetics and Breeding of Chili Pepper Capsicum spp.. In: Production and Breeding of Chili Peppers (Capsicum spp.). Springer, Cham. https://doi.org/10.1007/978-3-319-06532-8_4

25. Rukundo, P., Shimelis, H., Laing, M., Gahakwa, D. 2017. Combining ability, maternal effects, and heritability of drought tolerance, yield and yield components in sweet potato. Front. Plant Sci. 7, 1981.

26. Sprague, G.F., Tatum, L.A. 1942. General versus specific combining ability in single crosses of corn. Journal of the American Society of Agronomy. 34 : 923-932.

27. Supena, E.D.J., Custers, J.B.M. 2011. Refinement of shed-microspore culture protocol to increase normal embryos production in hot pepper (Capsicum annuum L.). Scientia Horticulturae. 130(4): 769-774.

28. Syukur, M., Sujiprihati, S., Yunianti, R. Undang. 2010. Diallel analysis using Hayman method to study genetic parameters of yield components in Pepper (Capsicum annuum L.). HAYATI Journal of Biosciences. 17(4): 183-188. http://journal.ipb.ac.id/index.php/hayati DOI: 10.4308/hjb.17.4.183.

29. Yadahalli, P., Srinivasa, V., Lakshamana, D., Hadapad, B., 2017. Combining ability studies for growth and yield characters Bell Pepper (Capsicum anuum L.). Enviroment and Ecology. 35(2): 1521-1525. 